\title{
Introduction to Non Commutative Algebraic Geometry
}

\section{Arvid Siqveland*}

Professor, Buskerud Vestfold University College

\begin{abstract}
Ordinary commutative algebraic geometry is based on commutative polynomial algebras over an algebraically closed field k. Here we make a natural generalization to matrix polynomial k-algebras which are non-commutative coordinate rings of non-commutative varieties.
\end{abstract}

Keywords: Non commutative algebraic geometry; Topology; Transition morphisms

\section{Introduction}

\section{Algebraic varieties}

In this introduction, we use Hartshornes classical book on algebraic geometry [2] as reference. We consider the free polynomial algebra over $k=\bar{k}$, Char $\mathrm{k}=0, \mathrm{~A}=\mathrm{k}\left[\mathrm{t}_{1} \ldots \mathrm{t}_{\mathrm{d}}\right]$. The affine $\mathrm{n}$-space is the set of points in $\mathbb{A}^{n}=k^{n}$, an algebraic set is given by an ideal $\mathfrak{a} \subseteq A$ as the zero set $Z(\mathfrak{a})=\left\{P \in \mathbb{A}^{n} \mid f(P)=0 \forall f \in \mathfrak{a}\right\}$. The algebraic sets are the closed sets in a topology on $\mathbb{A}^{n}$ called the Zariski topology, and an algebraic, affine variety is a closed, irreducible (i.e. it is not a union of two proper closed subsets, equivalently, every open subset is dense), subset of $V \subseteq \mathbb{A}^{n}$. One basic term in algebraic varieties is an arrow-reversing correspondence from closed subsets $Z(\mathfrak{a}) \subseteq \mathbb{A}^{n}$ to radical ideals $\sqrt{\mathfrak{a}} \subseteq A$. The ideal of a closed subset is $I(V)=\{f \in A \mid f(P)=0 \forall P \in V\}$

Thus $I(Z(V))=\sqrt{\mathfrak{a}}$, the radical of $\mathfrak{a}$.

There is a close connection between differential geometry and algebraic geometry, and because differential geometry is seen as a tool for applications (physics), the same is true for algebraic geometry. The topology in differential geometry is the smallest topology making the analytic functions continuous. In algebraic geometry, we work with polynomials rather that power-series, so we use the smallest topology that makes rational functions continuous. That is the Zariski topology defined above.

In the Zariski topology, we have the definition of regular functions: Let $U \subseteq \mathbb{A}^{n}$ be an open subset. A map $\Psi: u \rightarrow k$ is called regular if there exists polynomials $f, h$ such that $\psi(P)=\frac{f(P)}{h(P)}$ with $h(P) \neq 0$ for all $P \in U$.

Definition 1: The ring of regular functions defined over an open subset $U \subseteq V \subseteq \mathbb{A}^{n}$ is the ring $\mathcal{O}_{V}(U)=\{\psi: U \rightarrow k \mid \psi$ is regular on $U\}$, with its natural ring operations.

Definition 2: (Inductive and Projective Limits). A directed set ( $I$, $\leq$ ) is a partially ordered set $I$ such that every finite subset of elements has an upper bound, or equivalently, that for each pair $a, b \in I$ there is a $c \in I$ such that $a \leq c$ and $b \leq c$. Consider a small category $\mathbf{a}$.

a) A projective system of elements in $\mathbf{a}$ is a family of objects $\left\{A_{i}\right\}_{i \in I}$ together with transition morphisms $\psi_{i j}: A_{j} \rightarrow A_{i}$ for each pair $i \leq j \in I$ with the properties that, for each $i \in I, \psi_{i i}=i d$, and if $i \leq$ $j \leq k$ then $\psi_{i j}{ }^{\circ} \psi_{j k}=\psi_{i k}$. The projective limit of the projective system is defined as an object $\lim _{\leftarrow} A_{i} \in a$ a with morphisms $\psi_{i}: \lim _{i \in I} A_{i} \rightarrow A_{i}$

$$
\underset{i \in l}{\leftarrow}
$$

for each $i$ such that for all $i \leq j, \psi_{i}=\psi_{i j}{ }^{\circ} \psi_{j}$, and such that if $(Y, \phi)$ is another object with corresponding properties, then there is a unique morphism $\rho: Y \rightarrow \lim _{i \in I} A_{i}$ such that $\psi_{i}{ }^{\circ} \rho=\phi_{i}$. In a small category, to prove the unique existence of projective limits, we let

$$
\lim _{i \in I} A_{i}=\left\{a \in \prod_{i \in I} A_{i} \mid a_{i}=\psi_{i j}\left(a_{j}\right) \text { for all } i \leq j \in I\right\} .
$$

b) An Inductive system is the dual of a projective: It is a family of objects $\left\{A_{i}\right\}_{i \in I}$ together with transition morphisms $\psi_{i j}: A_{i} \rightarrow A_{j}$ for each pair $i \leq j \in I$ with the properties that, for each $i \in I, \psi_{i i}=i d$ and if $i \leq j \leq k$ then $\psi_{j k}^{\circ} \psi_{i j}=\psi_{i k}$.

The inductive limit of the inductive system is defined as an object $\lim A_{i} \in a$ with morphisms $\psi_{i}: A_{i} \rightarrow \lim A_{i}$ for each $i$ such that for all $i \leq j$

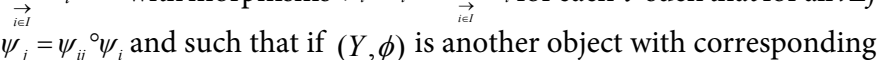
properties, then there is a unique morphism $\rho: \lim _{\rightarrow} A_{i} \rightarrow Y$ such that $\phi_{i}^{\circ} \rho=\psi_{i}$. In a small category, to prove the unique existence of inductive limits, we let

$$
\lim _{\vec{i} \in I} A_{i}=\coprod_{i \in I} A_{i} / \sim, a_{i} \sim a_{j} \Leftrightarrow a_{i}=\psi_{k i}\left(a_{k}\right), a_{j}=\psi_{k j}\left(a_{k}\right) \text { for some } k \geq i, j
$$

For the definitions in this text, we notice that the family of open subsets is a directed set partially ordered by inclusion. The ring of regular functions locally at $P$ is $\mathcal{O}_{V, P}=\lim _{U} \mathcal{O}_{V}(U)$, and by duality, we also have the other way around: $\mathcal{O}_{V}(U)=\lim _{\leftarrow} \mathcal{O}_{V, P}$.

By this we have that the coordinate ring of the variety $V$ is $\mathcal{O}_{V}(V) \simeq S(V)=A / I(V)$,

And that the ring of locally regular functions in $P$ is

$\mathcal{O}_{V, P} \simeq S(V)_{\mathfrak{m}_{P}}$

Where $\mathfrak{m}_{P}=I(P)=\left(t_{1}-P_{1}, \ldots, t_{d}-P_{d}\right)$ is the maximal ideal corresponding to $P$

*Corresponding author: Siqveland A, Dean, Faculty of Technology, Buskerud Vestfold University College, Kongsberg, Norway, Tel: +4731008918; E-mail: Arvid.Siqveland@hbv.no

Received February 02, 2015; Accepted April 11, 2015; Published April 21, 2015

Citation: Siqveland A (2015) Introduction to Non Commutative Algebraic Geometry. J Phys Math 6: 133. doi:10.4172/2090-0902.1000133

Copyright: (C) 2015 Siqveland A. This is an open-access article distributed unde the terms of the Creative Commons Attribution License, which permits unrestricted use, distribution, and reproduction in any medium, provided the original author and source are credited. 
The final definition of the category of affine varieties in the commutative situation is the definition of morphisms. Morphism between two affine varieties $V, W$ is a continuous map $\phi: V \rightarrow W$ such that the induced map $\phi^{\sharp}: \mathcal{O}_{W}(U) \rightarrow \mathcal{O}_{V}\left(\phi^{-1}(U)\right)$ is well defined for each open $U \subseteq W$, that is $f \mapsto f^{\circ} \phi$ is regular on $V$.

\section{Local Categories}

Everything in this section and the next can be found in $M$. Schlessinger's classical work [7]. Let $\ell$ denote the category of local artinian $\mathrm{k}$-algebras with residue field $\mathrm{k}$. That is diagrams

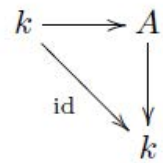

with A local, artinian. The morphisms in $\ell$ are the k-algebra homo morphisms commiting in the diagram. We let $\hat{\ell}$ denote the procategory, which is the category of projective limits in $l$. For any covariant functor $f: C \rightarrow$ Sets we have the following lemma:

Lemma 1 (Yoneda): For any object $C \in C$ there is an isomorphism

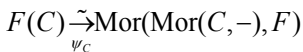

Given by $\psi_{C}(\xi)(A)(\phi)=F(\phi)(\xi)$, with inverse $\psi_{C}^{-1}(\phi)=\phi(C)(\mathrm{id}) \in F(C)$

The lemma extends to procategories, and is true for contra variant functors when we replace mor(C,-) with mor(-,C) . In particular:

Lemma 2: Let $f: l \rightarrow$ Sets be a covariant functor. Then for every $\hat{R} \in \ell$ there is an isomorphism

$$
\psi_{\hat{R}}: \hat{F}(\hat{R}) \rightarrow \operatorname{Mor}(\operatorname{Mor}(\hat{R},-), \hat{F})
$$

As usual $k[\varepsilon] \cong k[x] /\left(x^{2}\right)$ denotes the algebra of dual numbers. An epimorphism $\pi: R \rightarrow S$ in $l$ is called small if $\operatorname{ker} \pi \cdot \mathfrak{m}_{R}=0$ where $\mathrm{m}_{\mathrm{R}}$ is the maximal ideal in $R$. Finally, a transformation of functors $f, g: l \rightarrow$ Sets is smooth if for any small morphisms $R \rightarrow S$, in the diagram

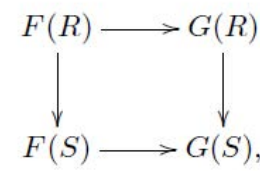

if objects $F(S) \ni x_{S} \mapsto y_{S} \in G(S)$ and $G(R) \ni y_{R} \mapsto y_{S} \in G(S)$, there is an object $x_{R} \in F(R)$ mapping to both $x_{S}$ and $y_{R}$

The following concept is the one we generalize in this text:

Definition 3: The couple $(\hat{R}, \hat{\xi})$ is said to prorepresent $F$ if $\psi_{\hat{\hat{R}}}(\hat{\xi})$ is an isomorphism. The couple is said to be a prorepresenting hull, or $\hat{R}$ is said to be a formal moduli with proversal family, $\hat{\xi}$, if $\psi_{\hat{R}}(\hat{\xi})$ is smooth and an isomorphism for $k[\varepsilon]$, (usually and reasonably) called the tangent level.

Lemma 3: A prorepresenting object is unique up to unique isomorphism. A prorepresenting hull is unique up to non unique isomorphism.

\section{Global to Local Theory}

Let $f: s c h k \rightarrow s$ Sets be a covariant functor. Assume there exists a fine moduli space for the set $\mathrm{F}(\mathrm{k})$ (which can be interpreted by the "family"-functor being representable). This means that there exists a scheme $\mathrm{M} / k$ and a universal family $u \in F(M)$ such that, with the notation above, $\psi_{\mathbb{M}}(\mathcal{U})$ is an isomorphism. Let $M \in F$ (Spec k) be an object represented by the closed point $[M] \in \mathbb{M}$, and define a covariant functor $F_{\ell, M} ; \ell \rightarrow$ Sets by

$$
F_{\ell, M}(R)=\left\{M_{R} \in F(\operatorname{Spec} R) \mid F(\operatorname{Spec} k \rightarrow \operatorname{Spec} R)\left(M_{R}\right)=M\right\} .
$$

Because $M$ is a fine moduli, $\operatorname{Mor}(-, \mathbb{M}) \simeq F \Rightarrow \operatorname{Mor}\left(\widehat{\mathcal{O}}_{\mathbb{M},[M]},-\right) \simeq \hat{F}_{\ell, M}$. This says that $\left(\widehat{\mathcal{O}}_{\mathbb{M},[M]}, \widehat{\mathcal{U}}_{[M]}\right)$ prorepresent $\hat{F}_{\ell, M}$ and so is unique up to unique isomorphism.

We call $F_{\ell, M}$ the local deformation functor. The idea is the following:

The local formal moduli represent the local, completed rings of the moduli scheme, and can be used to analyse, or to construct, the moduli scheme.

\section{Algebraic Varieties Revisited (Defined by local theory)}

Let $A=k\left[t_{1}, \ldots, t_{d}\right] / \mathfrak{a}$ be a $k$-algebra. Then Spec $A$ is fine moduli for its closed points (maximal ideals). A point $\mathfrak{m} \in A$ Spec A corresponds to a unique morphism $\phi_{\mathrm{m}}: \operatorname{Spec} k \rightarrow \operatorname{Spec} A$, i.e.

$\operatorname{Hom}(\operatorname{Spec} k, \operatorname{Spec} A) \simeq \operatorname{Pts}(\operatorname{Spec} A)$.

Definition 4: Let $M$ be an $A$-module. Then $D e f_{M}: \ell \rightarrow$ Sets is defined by

$$
\operatorname{Def}_{M}(S)=\left\{S \otimes_{k} A \text {-modules } M_{S} \mid M_{S} \text { is S-flat, } \mathrm{k} \otimes_{\mathrm{S}} \mathrm{M}_{\mathrm{S}} \simeq \mathrm{M}\right\} / \sim,
$$

where two deformations are equivalent if there is an isomorphism $M_{S} \rightarrow M_{S}^{\prime}$ commuting with the fibre, i.e.

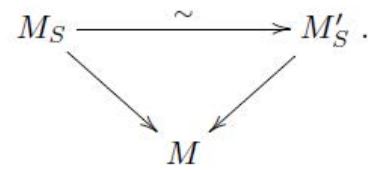

The earlier discussion shows that if $M=A / \mathfrak{m}$ for $\mathfrak{m} \subset A$ maximal, then $\hat{A}_{\mathrm{m}}$ pro represents $D e f_{A / m}$. Thus the affine theory can be defined as before, but with the local rings replaced by local formal moduli in each point. This is, by the way, the way we use deformation theory to construct moduli.

Notice that we have an injection $A \stackrel{\imath}{\rightarrow} \hat{H}_{A / \mathrm{m}}$ by definition, because an $H \otimes_{k} A$-structure on $A / \mathfrak{m}$, at over $\mathrm{S}$, is a homo morphism $A \rightarrow \operatorname{End}_{H}\left(H \otimes_{k} A / \mathfrak{m}\right) \simeq H, A \simeq i m \imath$.

\section{Non Commutative Affine Algebraic Geometry}

For the ordinary, commutative affine algebraic geometry, the basic object is the polynomial algebra in $d € N$ variables. In the non commutative situation, we take the matrix polynomial algebra as our basic object. That is:

Let $D=\left(d_{i j}\right) \in M_{r}(\mathrm{~N})$ be an $r \times r$-matrix. Then the matrix polynomial algebra $k[D]$ is the $r \times r$ matrix polynomial algebra generated by the idempotents $e_{i}, 1 \leq i \leq r$ together with the matrix variables $t_{i j}(1), \ldots, t_{i j}\left(d_{i j}\right)=t_{i j}$ for $1 \leq i, j \leq r$. We use the notation

$$
k[D]=\left(\begin{array}{ccccc}
k\left[\underline{t}_{1}\right] & \left\langle\underline{t}_{2}\right\rangle & \cdots & \left\langle\underline{t}_{(r-1)}\right\rangle & \left\langle\underline{t}_{(r)}\right\rangle \\
\left\langle\underline{t}_{21}\right\rangle & k\left[\underline{t}_{2}\right] & \cdots & \left\langle\underline{t}_{(r-1)}\right\rangle & \left\langle\underline{t}_{(r)}\right\rangle \\
\vdots & \vdots & \ddots & \vdots & \vdots \\
\left\langle\underline{t}_{r 1}\right\rangle & \left\langle\underline{t}_{r 2}\right. & \cdots & \left\langle\underline{t}_{r(r-1)}\right\rangle & k\left[\underline{t}_{r r}\right]
\end{array}\right) .
$$


Notice that we use the commutative polynomial $k$-algebras on the diagonal. This is not the natural free object in the category, but we use it because it is simpler to give a (naive) geometric interpretation.

Also notice that this notation implies that the multiplication of $t_{i j}(l)$ 's are given by matrix multiplication.

Example 1: $A=\left(\begin{array}{cc}k\left[t_{11}(1), t_{11}(2)\right] & k t_{12} \\ k t_{21}(1)+k t_{21}(2) & k\left[t_{22}(1), t_{22}(2)\right]\end{array}\right)$

Differential geometry was generalized to noncommutative geometry by Connes and Marcolli [3], and further developed by Dubois-Violette in [4].

Generalization of differential geometry to matrix algebras is given by Dubois-Violette, Kerner and Madore in [5].

We use results from the above referred articles in the generalization of algebraic geometry. Before we are ready to define the noncommuative analogue of the ring of dual numbers:

Definition 5: The non commutative $r \times r k$-algebra of dual numbers, also called the test algebra, is the algebra $T=k[D] / \mathrm{m}^{2}$

where $D$ is the $r \times r$-matrix with 1 in every entry, and $m$ is the ideal generated by all the variables $t_{i j}$.

The rest of the results in this section can be found in the work of Arnfinn Laudal [6].

Definition 6: The category $a_{r}$ is the category with objects Artinian algebras fitting in the diagram

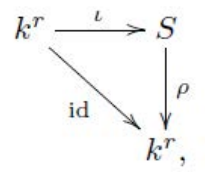

and such that $I^{n}(S):=\operatorname{ker}^{n} \rho=0$ for some $n \in \mathbb{N}$, and with morphisms the$\mathrm{K}^{\mathrm{r}}$-algebra homo morphisms commuting with the above diagrams.

Definition 7: Let $\mathcal{M}=\left\{M_{1}, \ldots, M_{r}\right\}$ be a set of $r \in \mathbb{N}$ right $A$ -modules, and put $M=\oplus_{i} M_{i}$ Then we define $\operatorname{Def}_{M}: \mathrm{a}_{r} \rightarrow$ Sets by

$$
D e f_{M}(\mathrm{~S})=\left\{S \otimes_{k} A-\bmod M_{S} \mid k^{r} \otimes_{S} M_{S} \simeq M, M_{S} \simeq_{S} S \otimes_{k^{r}} M\right\} / \cong,
$$
the relation $\cong$ being the one corresponding to the commutative situation. We must assume $M_{S}$ to be an $s-a$ abi module on which $k$ acts centrally.

Notice that the property $M_{S} \simeq_{S} S \otimes_{r^{r}} M$ says that the isomorphism is as $S$-modules. This is equivalent to $M_{S}$ being $S$-flat, but we take that into the definition.

Definition 8: $H_{M}$ is called the semilocal formal moduli with formally versal family $M$ if $\phi_{H_{M}}(\mathcal{M})$ is smooth, and an isomorphism for the test-algebra.

Lemma 4: The non commutative deformation functor $\operatorname{Def}_{M}: \mathrm{a}_{r} \rightarrow$ Sets has a semi local moduli determined by some welldefined Generalized Massey Products. That is to say, it can be constructed. Also, the construction gives a well-defined injection [6].

$$
A \rightarrow \operatorname{End}_{H_{M}}\left(H_{M} \otimes_{k^{r}} M\right) \text {. }
$$

Proof: See Eriksen [1] or Siqveland [8] for a constructive proof. A proof of existence can be given by generalizing the classical proof of Schlessinger in [7] verbatim.

Now we have all the needed tools necessary to define the noncommuative affine space.

Definition 9: Consider a matric polynomial algebra $A=k[D]$, $D=\left(d_{i j}\right) \in M_{r}(\mathbb{N})$. The affine algebraic space $\mathbb{A}^{D}$ of this algebra is the disjoint union of the affine spaces on the diagonal, that is $\mathbb{A}^{D}=\coprod^{r} \mathbb{A}^{d_{i j}}$, with the product (Zariski) topology. Each (closed) point in this space corresponds to a maximal ideal on the diagonal in the matrix algebra, which again corresponds to one-dimensional representations of $A$. For each finite set of (closed) points $V=\left\{V_{1}, \ldots, V_{s}\right\} \subset \mathbb{A}^{D}$, we let $V=\sum_{i=1}^{s} V_{i}$, and we define the semi local ring of $\mathbb{A}^{D}$ in $V$ as $\mathcal{O}_{\mathbb{A}, V}=\operatorname{End}_{H_{V}}\left(H_{V} \otimes_{k^{r}} V\right) \simeq H_{V}$.

The generalized concept of localization immediately gives the natural generalizations of affine varieties, regular maps, and morphisms. A lot of result needs to be established, which we will do in forthcoming work. Also, the deformation theory can be removed from the discussion, by defining the semi-local rings by their generalized Massey Products which can be given intrinsic.

Also, as algebraic geometry can be seen as a simplification of differential geometry for physical models, the noncommutative theory is needed for physical models involving entanglement.

For more examples, see the author's articles [9-11] where more examples appear as resulting algebras of noncommutative deformation theory.

\section{Acknowledgement}

I would like to thank Daniel Larsson and Olav Imenes for the help and discussion in the development of this naive theory. Of course, nothing could have been written without Eivind Eriksens thorough cooperation, and Arnfinn Lau-dal's main conducting. Also, I would like to thank the anonymous referees for corrections and suggestions improving the article.

\section{References}

1. Eriksen E (2006) An Introduction to noncommutative Deformations of Modules Lect Notes Pure Appl Math 243: 90-125.

2. Hartshorne R (1977) Algebraic geometry Graduate Texts in Mathematics. (First Edition), New York.

3. Connes A, Marcolli M (2007) Noncommutative Geometry, Quantum Fields and Motives. Colloquium Publications, American Mathematical Society, USA.

4. Dubois-Violette M (1997) Some aspects of noncommutative differential geometry. Contemporary Mathematics 203: 145-157.

5. Dubois-Violette M, Kerner R, Madore J (1990) Non-commutative differential geometry of matrix algebras. J Math Phys 31: 316-322.

6. Laudal OA (2003) Noncommutative Algebraic Geometry. Rev Mat Iberoamericana 19: 509-580.

7. Schlessinger M (1968) Functors of Artin rings. Trans-Amer Math soc 130: 208222

8. Siqveland A (2008) Standard example in noncommutative deformation theory. J Gen Lie Theory Appl 2: 251-255.

9. Siqveland A (2009) On moduli spaces of 3d Lie algebras. J Gen Lie Theory Appl 3: 61-75.

10. Siqveland A (2010) Group actions, orbit spaces and noncommutative deformation theory. Proceedings of the Estonian Academy of Sciences 59: 364-369.

11. Siqveland A (2011) Geometry of noncommutative k-algebras. J Gen Lie Theory Appl 5: 1-12 